\title{
Evaluation of the Facial Paralysis Degree
}

\author{
Junyu Dong1, Qianqian Wang1, Shengke Wang1 and Li' an Liu ${ }^{2}$ \\ ${ }^{1}$ College of Information Science and Engineering, Ocean University of China \\ ${ }^{2}$ Qingdao Haiser Medical Centre \\ China
}

\section{Introduction}

The majority research into human faces in computer vision and pattern recognition is concerned with normal people, i.e. their faces are not affected by any diseases and appear symmetric or approximately symmetric. However, a great number of people in the world are suffering from facial paralysis. Facial Paralysis is a devastating disorder. It is caused when the facial nerve, which sends nerve impulses to the muscles of the face, loses its function. Facial paralysis results in significant psychological and functional disability from the impairment of facial expression, communication and eye protection. For example, it usually affects one side of the face, causing a drooping mouth, drooling, and excessive tearing from one eye.

Thus, the evaluation of the degree of paralysis is very important (Beurskens \& Heymans, 2003), as different degree requires different medical treatment. For example, as a reliable and effective method, acupuncture doctors may use different needles based on the patient's paralysis degree. In the past, several evaluation criterions regarding face nerve were proposed. Traditional assessment of facial paralysis is by the House-Brackmann(HB) grading system (House \& Brackman, 1985) which was proposed in 1983 and has been adopted as the North American standard for the evaluation of facial paralysis. Grading is achieved by asking the patient to perform certain movements and then using clinical observation and subjective judgment to assign a grade of palsy ranging from grade I (normal) to grade VI (no movement). The advantages of the HB grading scale are its ease of use by clinicians and that it offers a single figure description of facial function. The drawbacks are that it relies on a subjective judgment and it is insensitive to regional differences of function in the different parts of the face.

To provide physicians with an objective and quantitative measurement of single-sided facial paralysis, several computer-based methods have been proposed. Maximum static response assay (MSRA) assesses facial function by measuring the displacement of standard reference points of the face (Johnson et al., 1994). The method measures the amplitude of standard facial movements by comparing facial photographs taken at rest and at maximum contraction. For voluntary expressions of a patient, Wang et al present a facial paralysis grading approach based on measuring the patient's asymmetry (S et al., 2004). Compared with other international grading scales for facial paralysis, such as House-Brackmann and DEFS, the advantages of the approach are that it is objective and can diagnose facial paralysis automatically. 
This book chapter introduces two methods for evaluating the degree of facial paralysis. The first type is the computer-based realization of traditional medical methods, for example the House-Brackmann $(\mathrm{H}-\mathrm{B})$ method, mainly using image processing and pattern recognition techniques. In order to assess the degree of movement in the different regions of the face, the patients are first asked to perform five separate facial expressions, which are raising eyebrows, closing eye, screwing up nose, drumming cheek and opening mouth. The basic idea is to compare the differences between key points or regions in the two half sides of a face. Techniques involved in this type of methods include face detection, salient point or region detection, edge detection, clustering and machine learning schemes. This type of methods might require user interaction with the software system.

The second type of methods aims to automatically evaluate the facial paralysis and provide evaluation results without user intervention. Same as the first type of methods, patients are also asked to perform facial motions instructed by doctors. Salient regions and eigen-based methods are used to measure the asymmetry between the two sides of face and compare the expression variations between the abnormal and normal sides. Support vector machines are employed to produce the degree of paralysis. Both methods are tested on voluntary clinic patients. The strength and weakness of both methods are also presented.

\section{Estimating degrees of facial paralysis based on Harris corner detection}

First we describe the first one that is the computer-based realization of traditional medical methods, mainly using image processing and pattern recognition techniques. Our main goal is to find several key facial feature points and use these points to obtain the differences of motions between the two halves of the patient's face. These points include eye corners, nose corners and mouth corners. We first change the images into gray ones and then apply smoothing filters to the facial images so that noises can be reduced. This is because the images are unavoidably influenced by noises which make it difficult to obtain correct result. There are two purposes of filtering: 1. smoothing the regions that are not in edge region; 2. protecting edge regions. The median filter is an effective method to wipe out noise in images. In the next step, we apply integration projection on images to divide them into an eye region, a nose region and a mouth region. In each region, we use the Smallest Univalue Segment Assimilating Nucleus (SUSAN) algorithm and Harris corner detectors to locate feature points. Finally, we decide the paralysis degree by the combination of different scores derived from these points by comparing the two halves of the faces.

\subsection{Integration projection for region detection}

The integration projection method is based on the projection of an image into different directions. Let the processed image be $G(x, y)$, the size of which is $\mathrm{M} \times \mathrm{N}$. Then the function of integration projection is described as follows:

The horizontal integration projection:

$$
H(x)=\sum_{1}^{N} G(x, y)
$$

The vertical integration projection: 


$$
V(y)=\sum_{1}^{M} G(x, y)
$$

The two projections can be used to decide different regions.

\subsection{Edge detection}

Our previous experiments have shown that if we direct the corner detector on image, we may not obtain all the points as we wish. Thus, we first extract the edge in the image, and then apply the Harris corner detector.

We choose SUSAN edge detector (SM \& JM, 2007) to extract edges. The SUSAN operator has been widely implemented by using circle masks. The usual radius is 3 to 4 pixels, and the smallest mask considered is the traditional $3 \times 3$ mask. The 37 pixel circular mask is used in all feature detection experiments unless otherwise stated.

The mask is placed at each point in the image and, for each point, the brightness of each pixel within the mask is compared with that of the nucleus (the centre point). Originally a simple equation determined this comparison:

$$
c\left(\vec{r}, \vec{r}_{0}\right)=\left\{\begin{array}{l}
1 \text { if }\left|I(\vec{r})-I\left(\vec{r}_{0}\right)\right| \leq t \\
0 \text { if }\left|I(\vec{r})-I\left(\vec{r}_{0}\right)\right|>t
\end{array}\right.
$$

where:

$\vec{r}_{0}$ is the position of the nucleus in the two dimensional image;

$\vec{r}$ is the position of any other point within the mask;

$I(\vec{r})$ is the brightness of any pixel;

$t$ is the brightness difference threshold and $c$ is the output of the comparison.

This comparison is done for each pixel within the mask, and a running total $n$ of the outputs $(c)$ is expressed as:

$$
n\left(\vec{r}_{0}\right)=\sum_{\vec{r}} c\left(\vec{r}, \vec{r}_{0}\right)
$$

Next, $n$ is compared with a fixed threshold $g$, which is set to $3 n_{\max } / 4$, where $n_{\max }$ is the maximum value which $n$ can take. The initial edge response is created by using the following rule:

$$
R\left(\vec{r}_{0}\right)= \begin{cases}g-n\left(\vec{r}_{0}\right) & \text { if } n\left(\vec{r}_{0}\right)<g \\ 0 & \text { otherwise, }\end{cases}
$$

where $R\left(\vec{r}_{0}\right)$ is the initial edge response.

The algorithm as described above can produce good result. However, a much more stable and sensible equation that can be used for choosing $c$ is:

$$
c\left(\vec{r}, \vec{r}_{0}\right)=e^{-\left(\frac{I(\vec{r})-I\left(\vec{r}_{0}\right)}{t}\right)^{6}}
$$




\subsection{Corner detection}

We use Harris corner detector to detect corners. The Harris corner detector (C \& M, 1988) is a popular interest point detector due to its strong invariance to rotation, scale, illumination variation and image noise. The Harris corner detector is based on the local auto-correlation of a signal, where the local auto-correlation function measures the local changes of the signal with patches shifted by a small amount in different directions. A discrete predecessor of the Harris detector was presented by Moravec. Here the discreteness refers to the shifting of the patches.

Given a shift $(\Delta x, \Delta y)$ and a point $\left(x_{i}, y_{i}\right)$, the auto-correlation function is defined as:

$$
c(x, y)=\sum_{W}\left[I\left(x_{i}, y_{i}\right)-I\left(x_{i}+\Delta x, y_{i}+\Delta y\right)\right]^{2}
$$

where I denotes the image function and $\left(x_{i}, y_{i}\right)$ are the points in the $\mathrm{W}$ (Gaussian window) centered on $(x, y)$.

\subsection{Algorithms for locating feature points}

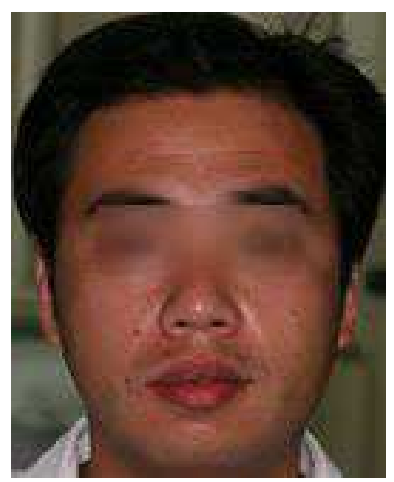

Fig. 1. An example image to be processed

\subsubsection{Locating eyes regions and points}

The location of eyes plays an important role in finding facial feature points. The following procedure can be used to locate eyes:

1. apply the Horizontal Integration Projection method on the image.

2. normalize the histogram of Integration Projection and find the y coordinate of the eyes according to minimum points of the histogram.

3. separate a region according to (2) and apply the Vertical Integration projection method to this region.

4. find two minimum points in the region and set them as the $x$ coordinate of center of eyes.

After locating the eyes, we find the eyes region according to the center point. Then we apply Susan edge detector to extract edge and use the Harris corner detector to locate eyes corner points. There are some points we do not want; we choose left-most, right-most, top and bottom points. 
Then we separate the eyebrow region based on the location of eyes. This is achieved by applying the Horizontal Integration Projection method on this region to find the $y$ coordinate of the eyebrows, and then applying the Vertical Integration Projection method to find the $x$ coordinate of the eyebrows.

Example results are shown in Fig. 2(a) and Fig. 2(b).

\subsubsection{Locating nose region and points}

Similar to locating eyes, the procedure for detecting the nose position is:

1. apply the Horizontal Integration Projection method to the region below the eye region;

2. find the first minimum point and set it as the y coordinate of the nose;

3. apply the Vertical Integration Projection to the region and set the height as [(y of nose)(y of eyes) ]*0.6;

4. find two minimum points and set them as the $x$ coordinate of the hole of nose;

5. separate the nose region;

6. apply the SUSAN edge detection method on the region to extract edges and use the Harris corner detector.

We choose the left-most and right-most points.

Example results are shown in Fig. 2(c).

\subsubsection{Locating the mouth region and points}

Similar to methods introduced in previous sections, we first apply integration projection to the region below the nose and set the first minimum point as $y$ of the mouth. According to this, we can find the mouth region. We use SUSAN method to extract edges and by using the Harris method we can find mouth corners and the middle line of the mouth. We choose the left-most, right-most and bottom points. The results are shown in Fig. 2(d).

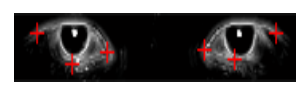

(a) eyes points

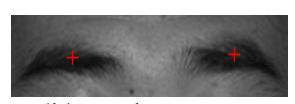

(b) eyebrow points

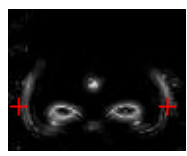

(c) nose points

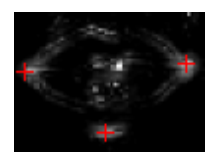

(d) mouth points

Fig. 2. Results of locating feature points

\subsection{The grading scheme}

We capture facial images of patients while they perform expressions. Fig. 3 shows facial feature points we need. The following expressions are required and the corresponding distances are measured:

1. closing eyes: the distance of $S_{0} I_{0}$ is calculated.

2. raising nose: the distance of $M_{c} L$ is calculated.

3. raising eyebrows: the distance of $S_{0} I_{0}$ is calculated.

4. plumping cheek: the distance of MMid is calculated.

5. opening mouth: the distance of MMid is calculated.

In order to avoid the limitation of subjective decision, Burres presents an objective decision system (Burres \& Fisch, 1986), namely linear measure index (B-FLMI), based on the research into calculating distances between certain points on human face. This system presents a 
method for recovery of face through measuring the percentage of distance (PD) of certain points. Although it can produce an objective evaluation of facial nerve, it is time consuming. In contrast, we apply face recognition techniques to evaluate facial condition.

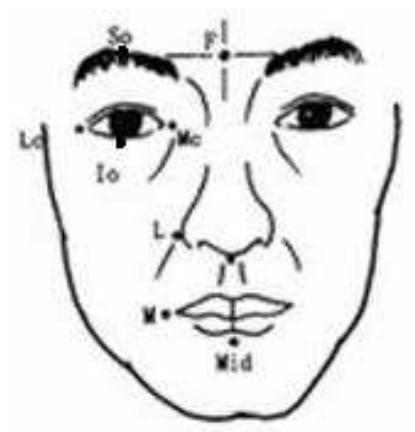

Fig. 3. Facial feature points (Junyu et al., 2008)

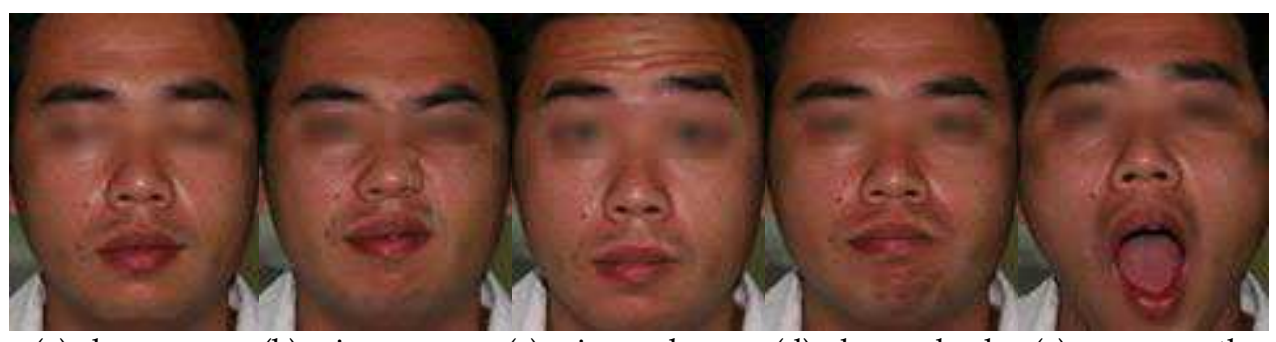
(a) close eyes
(b) raise nose
(c) raise eyebrow
(d) plump cheek
(e) open mouth

Fig. 4. Facial expressions made by a patient

The indices include:

1. two distance classes, i.e. $\mathrm{D}_{1}$ indicates the measured distances between designated points of the healthy half of face and $\mathrm{d}_{1}$ represents the distances between designated points on the sick half .

2. displacement percentage, i.e.

$$
\begin{aligned}
& P D=d_{1} / D_{1}, \text { if } D_{1}>d_{1} \\
& P D=D_{1} / d_{1}, \text { if } D_{1}<d_{1}
\end{aligned}
$$

3. $\mathrm{FNI}_{1} \sim \mathrm{FNI}_{5}$, which are indices of facial nerve function and denote the PD value of closing eyes, raising nose, raising eyebrow, plumping cheek, opening mouth.

4. the whole face nerve index, which is expressed as

$$
\text { TFNI }=(\text { sum of each FNI }) /(\text { number of indices })
$$

5. the percentage of face nerve motion function, which is defined by

$$
\operatorname{TPr}=(\text { unhealthy TFNI }) /(\text { healthy TFNI })
$$


This system divides motions mentioned above into regions as follows: forehead, eyes, nose and mouth. The global evaluation method can also be used for locating regions.

The establishment of two indices, TFNI and TPr, is based on the overlay ratio of diagnose of facial paralysis. TPr is the ratio of TFNI measured twice.

Based on the formula:

$$
T \operatorname{Pr}=T_{F N I} / T_{F N I}, T F N I=P D^{\prime} / P D
$$

We can obtain:

$$
T \operatorname{Pr}=\left(P D_{1} / P D_{2}\right) \times\left(P D_{2}{ }^{\prime} / P D_{1}^{\prime}\right)
$$

PD1/PD2 is the ratio of two measurements of the healthy parts. This ratio reduces the error caused by two measurements. Because patients go to see the doctor only after they are affected by facial paralysis, we normally cannot get healthy TFNI. Thus, we can only employ unhealthy TFNI. The result will be more precise if both the two indices are considered.

The following rules are used to produce the final conclusion:

1. if the value of TFNI is above 0.80 , we can decide that the patient may recover after 1 month.

2. if TPr is above 0.90 , there is more assurance.

3. if TPFI is $0.60 \sim 0.70$ and TPr is larger than 0.75 , the patient will recover in one year.

4. if TPr is $0.80 \sim 0.85$, they will recover in $2 \sim 3$ months.

If TFNI is less than 0.40 and TPr is less than 0.50 , the patient's face nerve is in bad condition and it should be treated early.

\subsection{Experimental results}

The experiments were carried out in a hospital. We ask 100 patients to perform certain facial expression and use the method introduced in the previous sections to evaluate the condition of patients. All images in our experiments were of frontal-viewed human faces and were warped to a standard size $(200 * 250)$. The results are well consistent with the diagnosis based on a group of doctor's decision. The accuracy is above $95 \%$. Table. 1 shows 10 example evaluation results. The final score is a weighted average of individual evaluation results.

\begin{tabular}{ccccccccccc}
\hline \hline & 1 & 2 & 3 & 4 & 5 & 6 & 7 & 8 & 9 & 10 \\
\hline FNI1 & 0.75 & 0.67 & 0.84 & 0.78 & 0.84 & 0.76 & 0.76 & 0.90 & 0.95 & 0.65 \\
FNI2 & 0.83 & 0.53 & 0.61 & 0.50 & 0.74 & 0.72 & 0.91 & 0.85 & 0.85 & 0.70 \\
FNI3 & 0.75 & 0.41 & 0.83 & 0.89 & 0.90 & 0.63 & 0.43 & 0.74 & 0.75 & 0.64 \\
FNI4 & 0.56 & 0.74 & 0.72 & 0.50 & 0.85 & 0.55 & 0.74 & 0.72 & 0.37 & 0.53 \\
FNI5 & 0.95 & 0.88 & 0.75 & 0.59 & 0.91 & 0.92 & 0.83 & 0.64 & 0.50 & 0.74 \\
TFNI & 0.77 & 0.65 & 0.75 & 0.65 & 0.85 & 0.72 & 0.73 & 0.77 & 0.68 & 0.65 \\
\hline \hline
\end{tabular}

Table 1. Examples of grading results. Each column shows the evaluation result for each facial expression. 


\section{Estimating degrees of facial paralysis based on salient point detection}

The key points introduced in the previous section can be seen as salient points, which are important for human visual system to capture key characteristics of an image. This section proposed another method for locating facial key points based on salient point detection.

In order to detect salient points quickly and accurately, we introduce a method based on wavelet transform. We first determine a set of key points based on salient point detection(Q et al., 2001), and then apply the K-MEANS clustering(K et al., 1998) algorithm to these key points and classify them into 6 categories. Finally, we calculate certain distances to estimate the state of facial paralysis.

\subsection{Salient point detection}

We apply a wavelet-based method for saliency detection to find the salient points which will include the key points we concern. The key points we concern include the two corners of the mouth, the topmost points of the two eyebrows, the internal and external corners of the eyes, the bottommost points of the eyes and the two points on the nosewing.

The wavelet representation of an image provides information about the variations in the image at different scales. We study the image at three scales $2^{j}, \mathrm{j}=-1,-2,-3$. At each scale, the image is decomposed into four sub-images. We use symbols LL, LH, HL and HH to represent approximate coefficients, horizontal details, vertical details and diagonal details respectively. In this paper, we denote the wavelet detail image as $W_{2}^{j} f$. We know from which signal points each wavelet coefficient at the scale $2^{j}$ is computed and we also calculate the coefficients at the scale $2^{j+1}$ at which the resolution is higher. There is a set of coefficients at the scale $2^{j+1}$ computed with the same points as a coefficient $W_{2}^{j} f(n)$ at the scale $2^{j}$. The set of coefficients is denoted as $C\left(W_{2}^{j} f(n)\right)$ and we call it the children of the coefficient $W_{2}^{j} f(n)$. Their relationship is as follows:

$$
C\left(W_{2^{j}} f(n)\right)=\left(w_{2^{j+1}} f(\kappa), 2 n \leq \kappa \leq 2 n+2 p-1\right), 0 \leq n \leq 2^{j} N
$$

where $p$ is the wavelet regularity and $N$ is the length of the signal $2^{-j} p$. A formula that can compute salient values to extract salient points is as follows:

$$
\begin{aligned}
& \text { saliency }=\sum_{\mathrm{k}=1}^{-\mathrm{j}}\left|C^{(k)}\left(W_{2^{j}} f(n)\right)\right| \\
& 0 \leq n \leq 2^{j} N,-\log _{2} N \leq j \leq-1
\end{aligned}
$$

We use the improved salient point detector based on wavelet transform instead of the above:

$$
\text { saliency }=\sum_{\mathrm{k}=1}^{-\mathrm{j}}\left|\mathrm{w}(k) C^{(k)}\left(W_{2^{j}} f(n)\right)\right|
$$




$$
0 \leq n \leq 2^{j} N,-\log _{2} N \leq j \leq-1
$$

Where $w(k)$ is the weight of the maximum wavelet coefficients at different scales. $w(k)$ is calculated according to the following set of expressions:

$$
\begin{gathered}
\mu_{\kappa}=\frac{1}{S} \sum_{z=1}^{S}\left|\mathrm{~W}_{2^{k}} f(z)\right| \\
\sigma_{\kappa}=\frac{1}{S} \sum_{z=1}^{s}\left(\left[\mathrm{~W}_{2^{k}} f(z)-\mu_{k}\right]^{2}\right)^{\frac{1}{2}} \\
w(k)=\frac{1}{\sigma_{k}}
\end{gathered}
$$

Where: $\mathrm{S}$ is the number of the set of maximum wavelet coefficients at $\mathrm{k}$ level:

$$
0 \leq z \leq s
$$

$\left|w_{2}^{\kappa} f(z)\right|$ is one of the elements of wavelet decomposition in the maximum coefficients.

A problem will appear when the patient's image contains closed eyes or mostly closed eyes. In this case, too many irrespective points can be found because of a great number of tiny wrinkles. Thus, the Log operator(D \& E, 1980) is applied to find edges of eyes. Next, we choose the SUSAN edge detector to extract edges. When edges are extracted, the points that are not on the edges are discarded from the set of salient points. The remaining set of salient points is mostly points about facial features and we denote it as $S$.

\subsection{K-MEANS clustering}

Salient points $\mathrm{S}$ detected as described in the above section gather in the regions of eyebrows, eyes, nose, mouth and the contour of the face. In order to find the 14 key points in the facial features separately, the salient points need to be classified into six categories. We apply the K-MEANS clustering algorithm to $S$ and classify them into 6 categories.

The main idea of K-MEANS is to define K centroids, one for each cluster. The steps are summarized as follows:

1. Select $\mathrm{K}$ points among the objects that are being clustered; these $\mathrm{K}$ points represent initial group centroids.

2. Assign each object to a group that has the closest centroid.

3. When all objects have been assigned, replace the K centroids with the new ones.

4. Repeat steps 2 and 3 until all the centroids do not change their positions any longer. The objects are separated into K groups finally.

\subsection{Key point detection}

We find the 14 key points on the edges of facial features respectively. The face model is shown as Figure 5:

We detect these key points among the salient points set $S$ found in the previous section according to the edges detected through the SUSAN edge detection algorithm. The procedures for finding the topmost points of the two eyebrows are summarized as follows: 


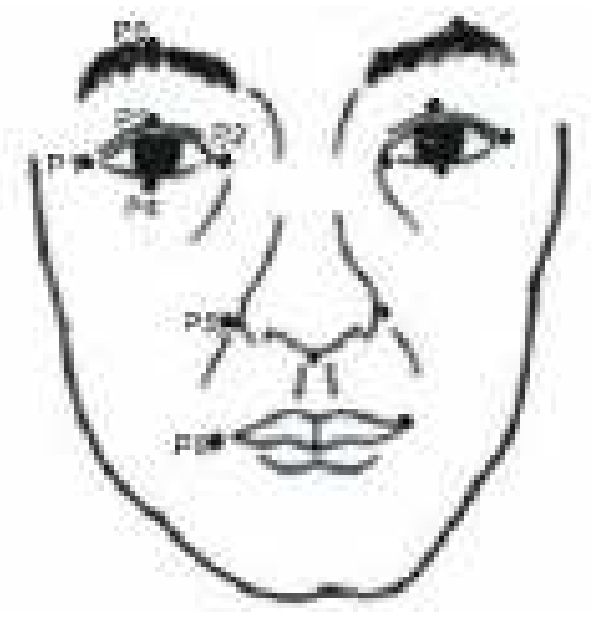

Fig. 5. Face model

We first select a topmost point from the salient point set of the right eyebrow and denote the selected point as $P_{0}$, then we check if the point $P_{0}$ is on the edge of the right eyebrow. If it is on the edge of the right eyebrow, then denote $\mathrm{P}_{0}$ as the topmost point of the right eyebrow. Otherwise, find a point $\mathrm{P}_{0 \text { new }}$ to replace $\mathrm{P}_{0} ; \mathrm{P}_{0 \text { new }}$ is calculated according to the following formulate:

$$
p_{\text {0new }}=\min \operatorname{imum}\left\{\left\|p_{0}-\chi_{i}\right\|^{2}\right\}
$$

where $x_{i}$ is any point in the salient points set of the right eyebrow.

The procedure for finding the other key points are similar to the steps of finding the topmost points of the two eyebrows. However, when the two topmost points of eyes are not accurate, the Log operator is applied to complement the deficiency. Edges of eyes are found using the Log operator and take place of the edges of eyes found using the SUSAN operator.

\subsection{The final estimation of the facial paralysis}

In this step, we apply the same method as introduced in the section of 3.5 to calculate the differences between the two sides of the face. The greater the difference is, the severer the disease is and the smaller the ratio is.

\subsection{Experimental results}

The images were taken in the hospital using the imaging set-up designed by ourselves. In our experiment we choose some patients to join our project voluntarily. We have stored their images which are divided into different classes according to different periods during the treatment. Each period has a final ration TPD obtained through our system. They are compared to ensure if the status of the patient is getting better, and the degree of the recovery. Our results are mostly consistent with the doctors' subjective evaluation.

Examples of results are shown in Figure 6 and Table 2: 


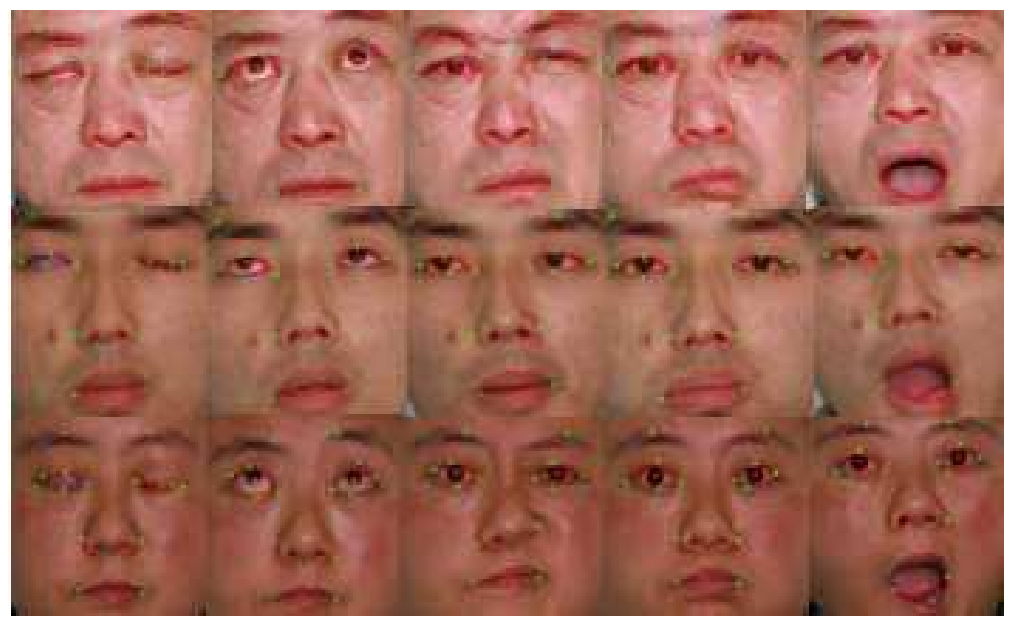

Fig. 6. Key points (green points: 14 key points)

\begin{tabular}{ccccccc}
\hline & PD1 & PD2 & PD3 & PD4 & PD5 & TPD \\
\hline 1 & 0.2678 & 0.7053 & 0.6274 & 0.8585 & 0.9090 & 0.67 \\
2 & 0.2666 & 0.9220 & 0.8415 & 0.9529 & 0.9779 & 0.79 \\
3 & 0.1250 & 0.8024 & 0.8150 & 0.6986 & 0.8429 & 0.66 \\
4 & 0.3000 & 0.7346 & 0.3837 & 0.7432 & 0.9687 & 0.63 \\
5 & 0.6875 & 0.7532 & 0.9259 & 0.9677 & 0.9863 & 0.86 \\
6 & 0.6521 & 0.8000 & 0.5416 & 0.9200 & 0.9602 & 0.77 \\
7 & 0.6470 & 0.9130 & 0.7246 & 0.8947 & 0.9834 & 0.83 \\
8 & 0.7325 & 0.9863 & 0.6962 & 0.7088 & 0.8564 & 0.80 \\
9 & 0.3042 & 0.8002 & 0.6901 & 0.8485 & 0.8746 & 0.70 \\
10 & 0.8974 & 0.9687 & 0.8754 & 0.9875 & 0.8759 & 0.92
\end{tabular}

Table 2. Some results

\subsection{Implement of the methods}

We implement the methods introduced in previous sections in a hospital. The whole system consists of software and an imaging device. First, we introduce the imaging set-up as shown in Figure 7. It includes a seat with a positioning device and a 3 million pixel camera. The imaging system has advantages that it consists of simple device, the manipulation is convenient and the estimation accuracy is high. Furthermore, it is an interactive and realtime device.

The software part of the system for facial paralysis evaluation is installed in a desktop PC and is connected with the image acquisition device. We can collect the images of patients' facial expression in real time and upload the images to the database. In the evaluation process, we can capture the same facial expression image repeatedly, until a satisfied image is obtained. 


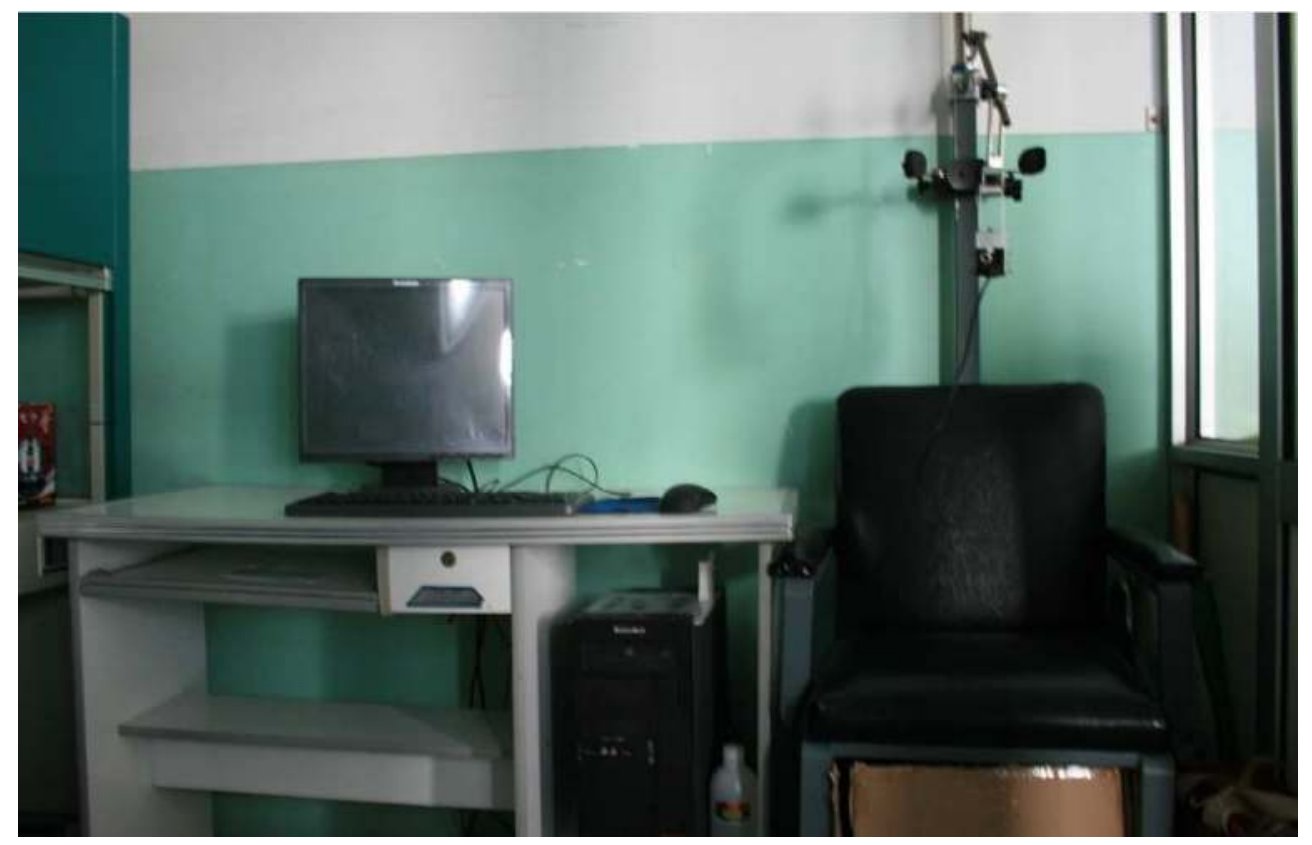

Fig. 7. The imaging system

So far the system has been tested based on evaluation of more than 120 patients. In the evaluation stage, we first load the patients' images from the camera. The system then automatically locates the key points which are in correspondence with every facial expression. If doctors think automatically located key points are not accurate enough, they can adjust the position of the key points manually. Finally, the system calculates a score according to the procedure introduced in the previous section. The score represents the degree of facial paralysis and is stored in the database for future reference.

\section{Conclusion and discussion}

While the majority research into human faces in computer vision and pattern recognition is concerned with normal people, we introduced methods for assessing degree facial paralysis 
for clinical purpose. Our main contribution is the proposition of a simple and efficient scheme for grading facial paralysis based on corner and salient point detection. The novel aspects include the location of the feature points and automatically evaluate the degree of facial condition. The proposed framework has produced promising results in clinical practice. The evaluation results produced by the methods are mostly consistent with the doctors' subjective assessment.

It should be noted that the two methods introduced in the previous sections have the same key problem, which can be described using intrinsic and extrinsic variability. The intrinsic variability in individuals can be due to multiple factors, including injury and age-related change. The extrinsic variability is also related to environment conditions and caused by viewing orientation, illuminations, shadows, and highlights. The variations of these factors can result in a significant decrease in facial recognition rate, and therefore make it difficult to detect key points. In this case, the software of implementation of the proposed methods requires the users' or doctors' intervention. To achieve a better performance, we elect to use the Active Appearance Model (AAM) (Cootes et al., 1998), which is well studied for face recognition, to model patients' faces due to facial paralysis. Our preliminary experiments have been carried out the results are promising for detecting key points and future work will include the use of AAM for the evaluation purpose.

\section{References}

Beurskens, C. H. G. \& Heymans, P. G. (2003). Positive Effects of Mime Therapy on Sequelae of Facial Paralysis: Stiffness, Lip Mobility, and Social and Physical Aspects of Facial Disability. Otology \& Neurotology, Vol.24, No.4, ( July 2003) , pp. 677-681

Burres, S. \& Fisch, U. (1986). The comparison of facial grading system. Arch Otolaryngol Head Neck Surg, (1986), pp. 753-758

C, H \& M, S. (1988). A combined corner and edge detector. Alvey Vision Conference, (1988), pp.147-151

D, M. \& E, H. (1980). Theory of edge detection. Proceedings of the Royal Society of London[C]. Vol. 207, No. 1167, (1980), pp. 187-217

House, J. \& Brackman, D. (1985). Facial nerve grading system. Otolaryngol Head Neck Surgery, (1985), pp. 146-147

Johnson, P. C., Brow, H., and Kuzon, W.M. (1994). Simultaneous quantification of facial movements: The maximal static response assay of facial nerve function. Annals of Plastic Surgery 5, (1994), pp.171-174

Junyu, D., Yang, L. and Li'An, L. (2008). An Approach to Evaluation of Degree of Facial Paralysis based on Image Processing and Pattern Recognition. Journal of Information System, Vol.5, No. 2, (2008), pp.639-646

K, A., S, R. and V, S. (1998). An Efficient k-means Clustering Algorithm. Proc. First Workshop High Performance Data Mining. (1998), pp.105-110

Q, T., N, S. and M.S.Lew. (2001). Image retrieval using wavelet-based salient points. Journal of Electronic Imaging. Vol. 10, No. 4, (2001), pp. 835-849

S, W., H, L., F, Q. and Y, Z. (2004). Objective facial paralysis grading based on $\mathrm{P}_{\text {face }}$ and eigenflow. Medical E Biological Engineering \& Computing, Vol.42 (2004) 
SM, S. \& JM, B. (2007). SUSAN-a new approach to low level image processing. International Journal of Computer Vision, Springer, Vol. 23, (2007), pp. 1573-1405

T. F. Cootes, G. J. Edwards and C. J. Taylor. (1998). Active Appearance Models. In ECCV98, (1998) 


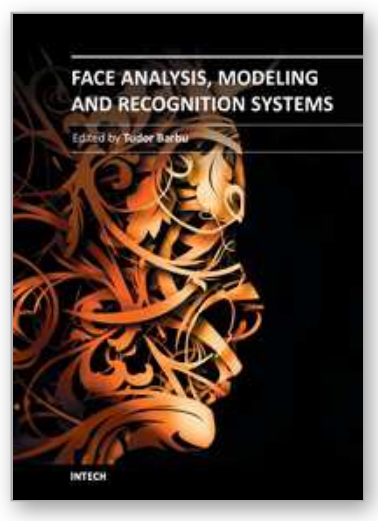

\author{
Face Analysis, Modeling and Recognition Systems \\ Edited by Dr. Tudor Barbu
}

ISBN 978-953-307-738-3

Hard cover, 212 pages

Publisher InTech

Published online 30, September, 2011

Published in print edition September, 2011

The purpose of this book, entitled Face Analysis, Modeling and Recognition Systems is to provide a concise and comprehensive coverage of artificial face recognition domain across four major areas of interest:

biometrics, robotics, image databases and cognitive models. Our book aims to provide the reader with current state-of-the-art in these domains. The book is composed of 12 chapters which are grouped in four sections. The chapters in this book describe numerous novel face analysis techniques and approach many unsolved issues. The authors who contributed to this book work as professors and researchers at important institutions across the globe, and are recognized experts in the scientific fields approached here. The topics in this book cover a wide range of issues related to face analysis and here are offered many solutions to open issues. We anticipate that this book will be of special interest to researchers and academics interested in computer vision, biometrics, image processing, pattern recognition and medical diagnosis.

\title{
How to reference
}

In order to correctly reference this scholarly work, feel free to copy and paste the following:

Junyu Dong, Qianqian Wang, Shengke Wang and Li'an Liu (2011). Evaluation of the Facial Paralysis Degree, Face Analysis, Modeling and Recognition Systems, Dr. Tudor Barbu (Ed.), ISBN: 978-953-307-738-3, InTech, Available from: http://www.intechopen.com/books/face-analysis-modeling-and-recognition-systems/evaluationof-the-facial-paralysis-degree

\section{INTECH}

open science | open minds

\section{InTech Europe}

University Campus STeP Ri

Slavka Krautzeka 83/A

51000 Rijeka, Croatia

Phone: +385 (51) 770447

Fax: +385 (51) 686166

www.intechopen.com

\section{InTech China}

Unit 405, Office Block, Hotel Equatorial Shanghai

No.65, Yan An Road (West), Shanghai, 200040, China

中国上海市延安西路65号上海国际贵都大饭店办公楼 405 单元

Phone: +86-21-62489820

Fax: $+86-21-62489821$ 
(C) 2011 The Author(s). Licensee IntechOpen. This chapter is distributed under the terms of the Creative Commons Attribution-NonCommercialShareAlike-3.0 License, which permits use, distribution and reproduction for non-commercial purposes, provided the original is properly cited and derivative works building on this content are distributed under the same license. 